\title{
Incidencia de infecciones del orificio de salida del catéter de hemodiálisis: cura con ciprofloxacino vs clorhexidina
}

\author{
José Luis Cobo Sánchez, Raquel Pelayo Alonso, Lucía Merino González, Ma Yolanda Vicente Jiménez, \\ Violeta Olalla Antolín, Magdalena Gándara Revuelta
}

\section{Hospital Universitario Marqués de Valdecilla. Santander}

\section{Introducción:}

Las guías de acceso vascular de las diferentes Sociedades Nefrológicas recomiendan el uso de clorhexidina $2 \%$ como antiséptico para curar el orificio de salida (OS) de los catéteres de hemodiálisis, con un grado de evidencia B(1-3). Estas guías desaconsejan el uso de soluciones antibióticas tópicas por el riesgo de crear resistencias y de infecciones por hongos. Sin embargo, la guía de Prevención de Infecciones Relacionadas con el Catéter de los CDC Norteamericanos, en su edición del 2011(4), señalan como excepción los catéteres de hemodiálisis, en los que si recomiendan el uso de soluciones antibióticas tópicas para la cura del $0 \mathrm{~S}$, con un grado de recomendación 1B.

\section{Objetivo:}

Comparar la incidencia de infección del OS del catéter de hemodiálisis entre la cura con ciprofloxacino en solución y con clorhexidina de base acuosa al $2 \%$.

\section{Metodología:}

Estudio cuasiexperimental con recogida de datos tras cada periodo de estudio. Criterios de inclusión: pacientes portadores de un catéter venoso central tunelizado que se sometieran a hemodiálisis crónica en nuestra unidad durante el periodo de estudio. Periodos de estudio: durante 6 meses (Enero- Junio 2012) se curaron los OS con ciprofloxacino en solución (Septociproß ótico $1 \mathrm{mg}$ ), y durante 6 meses (Julio-Diciembre 2012) con clorhexidina de base acuosa al $2 \%$. Durante ambos periodos la cura del OS se realizó bajo las mismas con- diciones: uso de medidas universales de asepsia, limpieza con suero salino fisiológico, secado con gasas estériles, aplicación de la solución de desinfección a estudio y cubierta con apósito de gasa autoadhesivo. Se definió infección del OS como un cultivo positivo de frotis de pericatéter junto con presencia de eritema, exudado (hemático, serohemático o purulento) y/o costra. La enfermera en cada cura valoraba la presencia de estos signos y si aparecían se recogía un cultivo. Se recogieron datos de la historia clínica del paciente (edad, sexo, nefropatía, datos filiación catéter, clínica del OS, resultados cultivos frotis pericatéter). Se calculó la tasa de infección del OS como el número de episodios de infección del 0S/días totales de catéter insertado X 1000 . Para el análisis estadístico se emplearon los test de chi cuadrado y T de Student.

\section{Resultados:}

Durante el periodo de ciprofloxacino se estudiaron 27 pacientes y 30 durante el periodo de clorhexidina. No hubo diferencias estadísticamente significativas entre las características clínicas de los pacientes entre ambos periodos. En el periodo de cura con ciprofloxacino aparecieron 2 infecciones del OS (tasa 0,12/1000 días-catéter), frente a 8 infecciones del $0 \mathrm{~S}$ durante el periodo de cura con clorhexidina (tasa 0,38/1000 díascatéter) $(p=0,001)$. El microorganismo más común durante el periodo de clorhexidina fue el Staphylococcus coagulasa negativa (5 infecciones) y durante el periodo de ciprofloxacino los responsables fueron el Staphylococcus aureus y el Staphylococcus coagulasa negativa. Los signos de infección más comunes fueron: costra hemática (26\%), eritema (18\%), exudado seroso (13\%) y exudado purulento ( $13 \%$ ). 


\section{Conclusiones:}

Según nuestros resultados la cura con ciprofloxacino en solución presenta menor incidencia de infecciones del OS del catéter de hemodiálisis que la cura con clorhexidina acuosa $2 \%$.

\section{Referencias Bibliográficas}

1. Rodríguez Hernández JA, González Parra E, Gutiérrez Julian JM, Segarra Medrano A, Almirante B, Martínez MT et al. Guías SEN. Guía de acceso vascular en hemodiálisis. Nefrología 2005; 25(Supl 1): 64-92.
2. National Kidney Foundation. KDOQI Clinical Practice Guidelines and Clinical Practice Recommendations for 2006 Updates: Hemodialysis Adequacy, Peritoneal Dialysis Adequacy and Vascular Access. Am J Kidney Dis 2006; 48(suppl 1):S1-S322.

3. UK Renal Association. Fluck R and Kumwenda M. Clinical Practice Guidelines. Vascular access for haemodialysis. 5th Edition. [Consultado 2 Abril 2012]. Disponible en: http://www.renal.org/Libraries/Guidelines/Vascular_Access_for_Haemodialysis_-_FINAL_VERSION_-_05_January_2011.sflb. ashx. 